\title{
Crossing the Worm-Brain Barrier by Using Caenorhabditis elegans to Explore Fundamentals of Human Psychiatric Illness
}

\author{
Donard S. Dwyer \\ Departments of Psychiatry and Pharmacology, Toxicology and Neuroscience, LSU Health Sciences Center at \\ Shreveport, Shreveport, LA, USA
}

\section{Keywords}

Anorexia · Antidepressants · Antipsychotics .

Endophenotypes - Major depressive disorder .

Protophenotypes · Schizophrenia

\begin{abstract}
Endophenotypes and Research Domain Criteria (RDoC) represent recent efforts to deconvolute psychiatric illnesses into fundamental symptom clusters or biological markers more closely linked to genetic influences. By taking this one step farther, these biomarkers can be reduced to protophenotypes - endophenotypes conserved during evolution - with counterparts in lower organisms including Caenorhabditis elegans and Drosophila. Striking conservation in C. elegans of genes that increase the risk for mental illness bolsters the relevance of this model system for psychiatric research. Here, I review the characterization of several protophenotypes that are relevant for asociality, avolition/anhedonia, prepulse inhibition, and anorexia. Interestingly, the analogous behavioral defects in C. elegans are also corrected by psychotropic drugs used to treat the corresponding symptoms in man and/or are mediated by the same neurotransmitters. Overall, there is much we can learn about the complex human brain by studying simpler nervous systems directing evolutionarily conserved behaviors. The potential for
\end{abstract}

generating important new insights from model organisms appears limitless when we begin to recognize the vestiges of evolution in ourselves.

(c) 2018 S. Karger AG, Basel

Psychiatric illnesses are multidimensional behavioral disorders that affect the most complex organ in man - the brain. Genetic contributions to the pathogenesis of schizophrenia, major depressive disorder (MDD), bipolar disorder, and other mental conditions are significant and well established. However, mutations in a single gene cannot account for the full spectrum of symptoms that characterize each of these disorders; variation in multiple genes must occur. Moreover, the complexity of the brain has hampered efforts to discover how genetic and environmental factors cause disease at a molecular level. To simplify the problem, researchers have broken down psychiatric illnesses into fundamental components (symptom clusters or domains) that are more closely linked to the effects of a single or limited number of genes. These simpler components have been termed endophenotypes - characteristic observable behaviors (e.g., prepulse inhibition [PPI] in schizophrenia) presumed to be modulated by specific genetic defects $[1,2]$. Here, I make the case for investigating protophenotypes - endophenotypes con-

\section{KARGER}

(c) 2018 S. Karger AG, Basel 
served during evolution - relevant for psychiatric illnesses in the model organism Caenorhabditis elegans.

Can model organisms reveal new information that helps us understand complex psychiatric disorders in man? At first glance, this seems unlikely because the evolutionary distance is so great and the structure of the nervous system of model organisms is too simple, e.g., there is no frontal cortex or limbic system. However, many behaviors essential for life have been conserved through evolution such as motivation to find food and mates and engaging in social activity. Furthermore, evolution requires that successful solutions to a problem crucial for survival (e.g., locating food) are selected rather than discarded and are embellished upon rather than reinvented to meet the needs of more advanced organisms in the evolutionary chain. This line of reasoning provides the logical framework for studying model organisms to learn about psychiatric illness, but is there any preliminary evidence that actually supports this notion?

Several intriguing observations encourage further investigation of simple model organisms in relation to psychiatry. First, Oliver Hobert [3] in a 2003 review referred to C. elegans mutant animals "hallucinating" the presence of food. This off-hand comment suggested that C. elegans might indeed experience false sensory signaling or misperceptions (sensing something that is not there) - the fundamental basis for hallucinations of any kind. Second, early studies by our group showed that antipsychotic drugs that cause weight gain in man induced lipid accumulation in $C$. elegans [4] indicating a conserved physiological response to psychiatric drugs. Finally, we searched the C. elegans genome for counterparts to schizophrenia risk genes identified in the largest genome-wide association study [5] and found that these genes were shared between nematode and man at a higher rate than genes in general. This suggests that many of the schizophrenia risk genes were conserved during evolution because they are involved in essential life processes common to animal species.

Previous articles have focused on the use of C. elegans to understand learning and memory [6], movement disorders [7], and drug addiction [8]. Little has been written about its utility for learning more about mental illness. This review will describe the use of $C$. elegans to model aspects of various psychiatric disorders, in particular schizophrenia, major depression, and anorexia nervosa (AN). The advantages and limitations of this approach will be discussed. Hopefully, the work highlighted here will inspire the creative use of model organisms to gain new insights into endophenotypes of psychiatric disorders at the molecular, genetic, and even circuitry-based level of resolution.

Using C. elegans to Explore Human

Psychiatric Illness

\section{Protophenotypes as Precursors of Endophenotypes}

Previously, protophenotypes were defined as the earliest form of an endophenotype that was conserved from primitive organisms, including mice, zebrafish, and C. elegans, to man [9]. Furthermore, to count as a protophenotype, a behavior should: (1) be regulated by conserved genes, even if those responsible have not been identified, (2) exhibit characteristic and relevant pathological features, and (3) be modified by drugs used to treat the human disorder associated with the corresponding endophenotype. The concept of protophenotypes is consistent with the idea of emotion primitives proposed by Anderson and Adolphs [10] to describe precursors of emotions shared across species. Darwin [11] articulated the original version of this argument by suggesting conservation of emotional expression from lower animals to man and recognition of these emotional states via similar features or phenotypes. While C. elegans may not experience paranoid delusions, animals can show defects in motivation and social interactions - major components of schizophrenia. Whereas worms may not be capable of feeling worthless or sad, they do show apathy and altered appetite - clinical features of MDD.

The concept of protophenotypes is consistent with the shift in research emphasis by NIMH toward Research Domain Criteria (RDoC) to define behavioral aspects of mental illness $[12,13]$. When symptoms of psychiatric disorders are reduced to the 5 domains, it is easier to see parallels for these domains and their associated constructs in model organisms. Based on this perspective, I will discuss 4 protophenotypes (summarized in Table 1) that appear to be relevant for schizophrenia, MDD, and eating disorders. Each protophenotype has one or more counterparts in RDoC domains: social feeding (Systems for social processes and Negative valence systems), immobility (Positive valence systems), startle suppression of pharyngeal pumping (Arousal/modulatory systems), and anorexic phenotypes (Negative valence systems).

\section{Social Withdrawal and Asociality versus Social Feeding}

Social withdrawal or asociality is a prominent negative symptom of schizophrenia [14]. A combination of genetic and clinical studies of schizophrenic patients has established asociality as an endophenotype that is also observed in close relatives $[15,16]$. In general, social behavior in social species is a delicate balance between

Mol Neuropsychiatry 2017;3:170-179 171 
Table 1. Summary of protophenotypes

\begin{tabular}{|c|c|c|c|c|}
\hline Endophenotype & Protophenotype & Genes & Neurotransmitters & Drugs (treat/induce) \\
\hline $\begin{array}{l}\text { Asociality/ } \\
\text { social withdrawal }\end{array}$ & Social feeding & $\begin{array}{l}n p r-1 \text { (NPY receptor) } \\
\text { daf-7 (TGF- } \beta \text { ) }\end{array}$ & $\mathrm{DA} ; 5-\mathrm{HT}$ & $\begin{array}{l}\text { Antipsychotics } \\
\text { Calmodulin antagonists } \\
\text { Gap junction inhibitors }\end{array}$ \\
\hline $\begin{array}{l}\text { Avolition/ } \\
\text { anhedonia }\end{array}$ & Immobility & $\begin{array}{l}\text { daf-2 (insulin/IGF-1 receptor) } \\
\text { unc-64 (syntaxin) }\end{array}$ & 5-HT; ACh & $\begin{array}{l}\text { Amitriptyline } \\
\text { Amoxapine } \\
\text { Cyproheptadine } \\
\text { Ritanserin } \\
\text { Metergoline } \\
\text { Clozapine } \\
\text { Olanzapine } \\
\text { Atropine } \\
\text { Pirenzepine }\end{array}$ \\
\hline Anorexia & Anorexic phenotype & $\begin{array}{l}\text { daf-16 (FOXO) } \\
\text { tph-1 (tryptophan hydroxylase) }\end{array}$ & DA; 5-HT & $\begin{array}{l}\text { Topiramate }(-) \\
\text { Acetazolamide }(-) \\
\text { Furazolidone }(-) \\
\text { Cyproheptadine }(+) \\
\text { Olanzapine }(+)\end{array}$ \\
\hline
\end{tabular}

competition where the individual gets ahead (more food, better habitat, etc.) and cooperation where the group prospers, e.g. safety in numbers against powerful predators. Due to this inherent tension, social behavior exhibits mixed valency with both positive and aversive aspects. In its pathological form in schizophrenia, asociality leads to anxiety in group situations, withdrawal from social interactions and suspiciousness of others.

Social interactions are widely observed in common model organisms, including Drosophila and C. elegans [17]. In C. elegans, the main example of social behavior is called social feeding characterized by aggregation of animals into clumps on food and bordering at the edge of a bacterial lawn $[18,19]$ as compared to solitary feeding of the wild-type N2 reference strain. Social feeding has previously been validated as a suitable protophenotype for social withdrawal based on the following findings [9]. (1) It is caused by a loss-of-function ( $l f)$ mutation in a gene (NPY receptor) conserved from C. elegans to man [18]. Interestingly, NPY levels are reduced in the brains of schizophrenic patients [20], and mice with the NPY Y2 receptor knocked out exhibit schizophrenia-like deficits [21]. (2) The social feeding phenotype is schizophreniarelevant in two areas. There is a significant correlation between CSF concentrations of NPY and ratings of social competence in schizophrenic patients [22]. Secondly, the behavior of $n p r-1(l f)$ animals is motivated by avoidance of perceived aversive conditions, which is consistent with the role of NPY as an anxiolytic agent in rodents $[23,24]$. (3) Drugs used to treat schizophrenia reduce social feeding in C. elegans (via tranquilizing effects?). This includes a broad spectrum of antipsychotic medications: chlorpromazine, haloperidol, fluphenazine, and olanzapine [9]. Fluphenazine is intriguing because it was used as an anxiolytic tranquilizer when first introduced into clinical practice.

Additional strains (e.g., CB1372 daf-7(e1372)) exhibit social feeding [19]. By contrast, the daf-7 mutation affects a TGF- $\beta$ family member involved in development and $\mathrm{CO}_{2}$ avoidance. This last behavior is notable because $n$ prl(lf) strains show enhanced $\mathrm{O}_{2}$ avoidance, which explains why they border due to lower $\mathrm{O}_{2}$ levels at this location on the lawn [25]. Aggregation would likewise reduce ambient $\mathrm{O}_{2}$ levels experienced by animals that form a clump. Thus, bordering and aggregation reflect aversive responses to a perceived threat - high $\mathrm{O}_{2}$ levels - and suggest a relative state of agitation or physical discomfort. In terms of $\mathrm{RDoC}$ constructs, social feeding involves negative va- 
lence systems related to potential threat (elevated levels of $\mathrm{O}_{2}$ ) and social processes (affiliation) to reduce ambient $\mathrm{O}_{2}$ concentrations. Thus, the social aspect of aggregation in C. elegans is not mediated via bonding or positive motivations (e.g., increased eating), but by avoidance and safety-in-numbers considerations.

The relevance of social feeding for social withdrawal is further supported by the neurochemistry involved. Dopamine and serotonin mediate the sense of threat and avoidance behavior, whereas drugs that inhibit aggregation block dopamine and serotonin receptors [9] - the main targets of antipsychotic drugs. Moreover, these same drugs inhibit social feeding in daf-7(e1372) animals [Dwyer, unpubl. obs.]. Of course, the neurochemistry and circuitry controlling social feeding cannot match the complexity of interactions in the brain stem-limbic system-frontal cortex that underlie social activity in man. Nevertheless, the predictive value of the model and its strong correlation with neurochemical defects in schizophrenia are very encouraging.

\section{Anhedonia, Apathy, and Avolition versus Immobility and Diminished Motivation}

Diminished motivation states constitute endophenotypes in both MDD and schizophrenia. In MDD, anhedonia and apathy are considered biological markers contributing to the overall picture of depression [26, 27]. In schizophrenia, avolition is considered an endophenotype that contributes to the negative symptoms [15, 28, 29]. In both cases, the most fundamental and shared feature is diminished motivation to either enjoy life or initiate productive activities of life.

Recently, a protophenotype for diminished motivation has been reported in C. elegans [30]. Mutant animals with temperature-sensitive loss of function in insulin/ IGF-1 signaling (IIS) via the insulin receptor (daf-2) become immobile when they experience acute food deprivation in the presence of low concentrations $(0.8-1 \%)$ of DMSO. A second mutation in syntaxin (unc-64), which regulates release of neuropeptides including insulin, produces a similar avolition/apathy phenotype. The animals are capable of moving in response to touch or exposure to aversive chemicals - they are not paralyzed. However, they fail to exhibit self-directed movement and remain in the same location on the plates until they die. Normally, acute food deprivation stimulates animals to increase their activity and commence distinctive foraging behavior to find food. By remaining immobile, animals over-

Using C. elegans to Explore Human

Psychiatric Illness ride instinctual programs that motivate survival behavior. In a very real sense, the worms are "suicidal" - they will die due to their inaction.

On the surface, the lack of self-directed behavior in IIS-deficient animals resembles either tonic immobility states induced by stress (e.g., "playing possum") and thus catatonia [31], or immobility in the forced swim test, which is an established model of depression and despair in rodents [32]. The main treatment for catatonia - benzodiazepines - failed to correct immobility, whereas antidepressants and certain second-generation antipsychotic drugs stimulated normal foraging [30]. Based on the pharmacology and the genetics of the response, this C. elegans protophenotype was considered more relevant for diminished motivation underlying the anhedonia and avolition characteristic of depressive states or negative symptoms. Alternatively, the immobility may reflect aberrant risk-reward calculations or effort allocation such that animals conserve effort rather than searching for food because they sense little chance for reward.

Avolition/apathy in the model is maintained by excessive muscarinic cholinergic and serotonergic signaling. This was confirmed by overcoming immobility with muscarinic receptor antagonists (e.g., atropine and pirenzepine) and 5-HT2 receptor-selective inhibitors (e.g., ritanserin and cyproheptadine). These findings provide support for the potential clinical relevance of this model because antimuscarinic agents (e.g., scopolamine [33]) and 5-HT2 receptor antagonists have both been used to treat depression $[34,35]$. The diminished motivation is also improved by certain antipsychotic drugs (clozapine and olanzapine) with superior efficacy against negative symptoms, including suicide attempts/ideation. Moreover, drugs that decrease immobility in rodents in the forced swim test likewise reduce immobility in C. elegans. Given the evolutionary distance between C. elegans and vertebrates, it is remarkable that these drugs produce the same effects on immobility.

The clinical significance of this protophenotype is further highlighted by the following observations. First, induction of immobility in C. elegans requires loss of function in IIS, which is linked to diabetes in man. Second, in patients diagnosed with diabetes, there is a 2- to 3 -fold increase in the incidence of depression [36], which includes anhedonia and apathy. Third, diabetic mice show increased immobility in the forced swim test and this is reversed by insulin [37]. Taken together, these findings provide supportive evidence for the idea that insulin plays an evolutionarily conserved role in mediating motivation. A scheme for how IIS may regulate neuronal activ- 


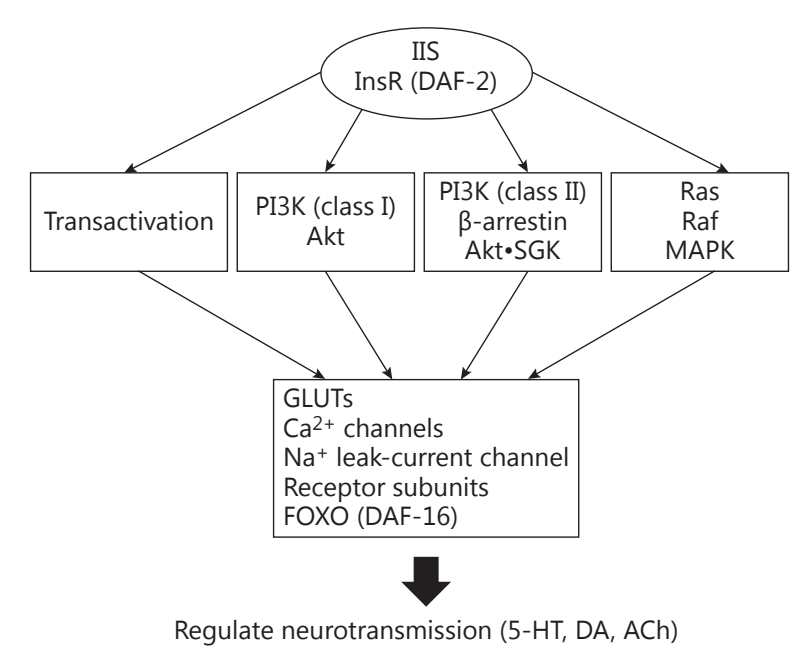

Fig. 1. Insulin/IGF-1 signaling (IIS) pathways regulate effectors of motivation. The insulin/IGF-1 receptor (InsR/DAF-2) signals by transactivating other cell surface receptors, activating phosphatidylinositol 3-kinases (type I and II) and the mitogen-activated protein kinase (MAPK) cascade. Downstream targets may be directly phosphorylated by activated kinases, such as Akt and serum- and glucocorticoid-inducible kinase (SGK), or indirectly affected by changes in protein turnover or gene expression mediated by FOXO. The potential downstream targets in neurons that mediate changes in motivation include various ion channels, transporters, and neurotransmitter receptors. Ultimately, IIS affects release and actions of major neurotransmitters such as serotonin and acetylcholine, which were implicated in studies of motivation in C. elegans [30].

ity is shown in Figure 1. It will be important in the future to confirm the pathways, fill in gaps in our knowledge and establish the circuitry involved.

\section{PPI and Exaggerated Startle versus Startle Inhibition of Pharyngeal Pumping}

A defect in sensorimotor gating revealed by the PPI task is considered to be a biological marker of schizophrenia $[38,39]$, although it is also found in individuals with other brain disorders [39]. PPI refers to the inhibition of a reflexive response (e.g., startle by a loud tone) when a prepulse (softer tone) is presented shortly before the startling stimulus. Schizophrenic patients and their close relatives show less inhibition than normal subjects on this task, i.e. less suppression of their startle response. In general, excessive startle and arousal is observed in people with schizophrenia.
The neurochemistry and circuitry underlying PPI has been extensively characterized in rodent models and in humans to a lesser degree. These studies reveal that dopamine, GABA, and glutamate are the major neurotransmitters involved $[38,40]$. PPI is mediated via stimulus processing in the brain stem and sensorimotor gating at the level of the midbrain with modulatory input from higher brain regions. The entire circuitry is referred to as the cortico-striatal-pallido-pontine system [39, and references therein].

The startle response is an ancient reflex that has been conserved through evolution [41]. Functionally equivalent behaviors are evident in mice, zebrafish, and C. elegans. One caveat concerns how closely startle in C. elegans mimics the startle response of mammals with the biggest difference being the potential for top-down modulation of reflexive behavior - an embellished function of more highly developed mammalian nervous systems. In C. elegans, touch on the tail with a wire pick briefly inhibits pharyngeal pumping, which is normally continuous at a high rate. This startle response is referred to as startle inhibition of pharyngeal pumping and represents a potential protophenotype for PPI. From the work of Keane and Avery [42], we know that GABA and glutamate regulate suppression of pharyngeal pumping in C. elegans. The pathway includes glutamate-activated $\mathrm{Cl}^{-}$channels whose closest homolog in humans is the glycine receptor (GLRA1). This gene in man has been implicated in an exaggerated excitability syndrome known as hyperekplexia characterized by excessive startle in response to mild stimuli and spasmodic stiffening of muscle [43]. Generalizations from simpler systems, such as this, help to validate the kind of behaviors that have been conserved during evolution.

Additional studies [Dwyer, unpubl. obs.] reveal that dopamine plays a major role in the regulation of startle inhibition of pumping in C. elegans. In the absence of dopamine, there is prolonged inhibition of pumping, which is consistent with the idea that failure to inhibit during PPI in schizophrenic patients is due to elevated dopamine levels. Furthermore, dopamine's actions in the C. elegans response require D2 dopamine receptors. This strengthens the pharmacological evidence for overlap between PPI and the startle inhibition response; however, this model is not a precise counterpart because there is no prepulse. Like PPI, startle inhibition in C. elegans is regulated by higher-order interneurons that integrate various signals including the fed state of the animal [42].

The similarities between PPI and startle inhibition extend beyond the neurochemistry and may provide in- 
sights into underlying neural circuitry - one of the goals of the RDoC reformulation of psychiatric illnesses. Neuronal connectivity in the $C$. elegans nervous system has been mapped in exquisite detail. To explain how touch on the tail communicates with pharyngeal neurons to inhibit pumping, a simplified neural circuit has been constructed (Fig. 2a). What has been termed a countercircuit is at the core of this scheme in order to explain the role of dopamine. The dopaminergic neurons ADE and CEP connect to each other and both express $\mathrm{G}_{\mathrm{oa}}{ }^{-}$ coupled D2 dopamine receptors. Although they share some common upstream inputs, they also receive synaptic signals from distinct sets of neurons. This countercircuit would operate in the following way. ADE neurons release dopamine, which acts via $\mathrm{D} 2$ dopamine receptors (DOP-2) on CEP neurons to suppress their activity. By contrast, when CEP neurons are active, they will release dopamine to suppress the ADE neurons. Each set of neurons modulates different outputs that are often contrary, for example, enhanced slowing of movement upon food encounter versus rapid foraging upon food deprivation. These behaviors are incompatible staying to eat versus leaving to find food - yet this simple counter-circuit can easily mediate both with an economy of components.

Human brain dopaminergic systems appear to display similar features as shown in Figure 2b. Three major dopaminergic pathways - nigrostriatal, mesolimbic, and mesocortical - are also arranged with collateral connections between pathways, expression of $\mathrm{D} 2$ receptors and incompatible behavioral outputs [44-47]. For example, dopamine in the mesolimbic pathway would promote emotional behavior, which might simultaneously suppress mesocortical activity, thereby suppressing higherorder planning and logical thought. Thus, a highly emotional state would be incompatible with higher-order thinking and vice versa due to the inherent properties of the putative counter-circuit proposed here. The simplicity of the C. elegans system may have allowed discernment of a fundamental circuit arrangement that has been conserved during evolution of a bigger brain. Of course, the neural circuitry-mediating emotion and logical thinking is far more complicated in the human nervous system than suggested here. Nonetheless, it may be informative to explore the role of similar dopaminergic counter-circuits in vertebrates and in relation to behavioral regulation and pathological states.

\section{AN and Restricted Caloric Intake versus Appetite Dysregulation and Anorexic Phenotypes}

Overexpression of forkhead box O (FOXO) genes in Drosophila produces an anorexic phenotype characterized by apparent ambivalence toward food, decreased food consumption, reduced foraging, and slower reproductive maturation [48]. A similar decrease in foraging has been observed when FOXO (DAF-16) activity is increased in C. elegans [49]. Elsewhere, the relevance of these anorexic phenotypes for the human eating disorder, AN, has been documented [50]. This work draws attention to biological, as opposed to psychosomatic, origins for AN. Guisinger [51] has speculated that survival during times of famine required a subpopulation of individuals who had reduced interest in food, increased activity levels upon starvation, and denial of harm. What evolved as a successful approach to deal with recurring famine is now considered a pathological state when it manifests in times of plentiful food. Furthermore, the biological response to starvation is regulated by factors such as FOXO and $5^{\prime}$ AMP kinase that have been conserved from yeast to man [52]. Consequently, protophenotypes in model organisms related to appetite regulation and foraging are likely to be relevant for corresponding behavioral features of AN.

To validate the use of model organisms to study AN, drugs that cause anorexia (loss of appetite) in man have been evaluated for their ability to suppress foraging in $C$. elegans. Topiramate, acetazolamide, and furazolidone have been reported to cause anorexia as a side effect, and all of these drugs significantly decrease food seeking behavior [Dwyer, unpubl. obs.]. By contrast, cyproheptadine, used as an appetite stimulant [53], and olanzapine, noted for causing weight gain in patients [54], both enhance foraging in C. elegans. These studies are a start in the right direction. Obviously, a C. elegans model of AN cannot fully address factors such as body dysmorphia, obsession about appearance, and fear of gaining weight. However, if the biology of the starvation response is a bigger driving force behind anorexic behavior than is assumed, then basic studies in model systems may yield a better understanding of AN than has emerged from current approaches.

\section{Psychiatric Drugs in C. elegans}

Simple model organisms, including C. elegans, have proven valuable for investigating mechanisms of action of psychotropic drugs and identification of new targets of these drugs [recently reviewed in 55]. In fact, it has been 


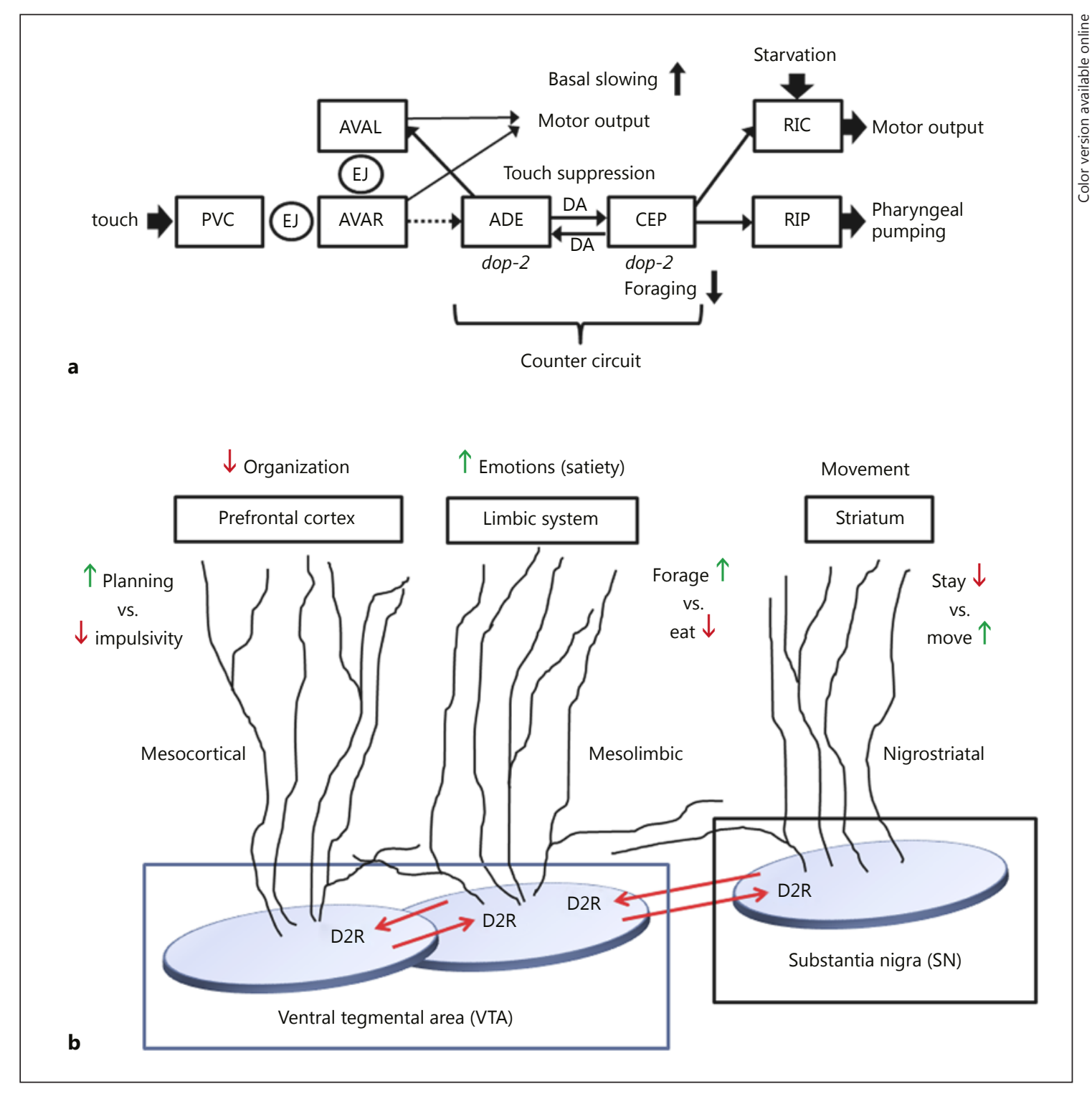

Fig. 2. Dopaminergic counter-circuit mediates opposing or contrary behaviors. a This scheme depicts neurons (three-letter designation in boxes) that connect the startle (touch) response to the only neuron, RIP, at the interface of the C. elegans head ganglia and the separate pharyngeal nervous system. The neuronal pathway that inhibits pumping has not been shown. To overcome startle suppression of pumping, the CEP neurons that innervate RIP must be inhibited. This is accomplished by dopamine (DA) released from ADE neurons acting through inhibitory D2 dopamine receptors (dop-2). The figure also shows how contrary behaviors, e.g. basal slowing vs. foraging, can be regulated by the counter-circuit. EJ refers to electrical or gap junctions between neurons. The dotted arrow from AVAR reflects an indirect connection to ADE mediated by additional neurons left out for simplicity. $\mathbf{b}$ Based on vari- ous studies [44-47], human brain dopaminergic pathways (labeled) appear to include counter-circuits in their neural architecture. The illustration represents a possible arrangement of local and trans-pathway collaterals that provide functional connections to regulate contrary behaviors. The striatum would mediate stay vs. move signals, whereas elements of the limbic pathway would promote foraging vs. eating. When the pathways act in concert, a person can either move and forage or stay and eat. Likewise, the mesocortical pathway can mediate planning and impulsivity in the prefrontal cortex, but not both at the same time. When one activity is high, dopamine release from that pathway will down-modulate the contrary activity. This arrangement is also a mechanism to fine tune ongoing activity in the primary pathway. 
both surprising and encouraging that so many drugs originally optimized to treat human psychiatric illness also show potent activity in C. elegans against disease-relevant behaviors. Moreover, these drugs show selectivity in their actions. For example, antidepressants correct immobility in C. elegans, whereas benzodiazepines do not [30]. Therefore, immobility may be more relevant as a model for depression than for catatonia or anxiety disorders. Similarly, haloperidol and trifluoperazine affect social feeding, but not immobility [9], which might indicate the relevance of social feeding for negative symptoms of schizophrenia but not depression.

C. elegans has already been instrumental for the identification of new targets and signaling pathways affected by CNS drugs [55]. For example, studies of clozapine revealed that it binds to the trace amine-associated receptor TAAR1, which was then implicated in clozapine enhancement of PPI [56]. Moreover, two independent groups simultaneously identified the IIS pathway in C. elegans as a physiologically relevant target of a wide variety of antipsychotic drugs $[57,58]$. This work discovered a novel mechanism for coactivation of the insulin receptor and identified a potential mechanism by which clozapine may induce diabetes insipidus and hypertension [59]. Thus, C. elegans can also be used to explore mechanisms related to the side effects of psychiatric drugs that may be the result of either unappreciated on-target effects or novel off-target ones.

In view of these various findings, it will be worthwhile to further evaluate the possibility that behaviors affected by psychiatric drugs in C. elegans are directly relevant for the human disorders treated with these same drugs. This line of thinking provided part of the original impetus for developing the concept of protophenotypes. By extension of this idea, what might lithium correct in C. elegans? Is the pathway or behavior affected relevant to bipolar disorder? Do anxiolytic agents affect any behavior in C. elegans? Responding to aversive threats (anxiety-inducing stimuli?) has certainly been shared across species, so there is the opportunity for relevance to anxiety disorders in man. Despite the obvious limitations to the use of C. elegans to inform us about human psychiatric illness cited throughout this review, the most serious restraint is simply the imagination of the investigator.

\section{Conclusions and Perspectives}

Genes implicated in genetic risk studies of psychiatric illness are largely conserved during evolution with clear homologs even in C. elegans. This finding suggests that

most of these genes are essential for life because they play critical roles in evolutionarily conserved behaviors, including appetite regulation, motivation, social interactions, threat avoidance, effort allocation, and associative learning based on rudimentary reward systems. In view of this conservation of genes and behavior, C. elegans, Drosophila, zebrafish, etc. represent complementary systems for modeling fundamental behaviors or protophenotypes. The deconvolution of complex psychiatric diseases into protophenotypes is consistent with the $\mathrm{RDoC}$ initiative and, in fact, most domains and constructs have equivalents in simple model organisms. Naturally, investigation of genes unique to man or higher vertebrates that are involved in psychiatric illness or behaviors with no counterpart in model organisms provides additional insights otherwise unobtainable. However, these insights are only possible because of comparisons with less advanced species. If worms can form social connections, hallucinate, act "suicidal," and show changes in motivation and appetite based on gene mutations and psychotropic drugs, there is every reason to include them in research into genetic and molecular mechanisms contributing to psychiatric illness in man. By stretching our imaginations to envision the thread connecting behavior in C. elegans to man, we may make discoveries that would be impossible with a more conventional and parochial mindset.

\section{Acknowledgements}

I would like to thank the Department of Psychiatry at LSU Health Sciences Center-Shreveport for financial support and Merve Kasap for helpful comments on the paper.

\section{Disclosure Statement}

I do not have any conflicts of interest to declare.

References

Mol Neuropsychiatry 2017;3:170-179 DOI: $10.1159 / 000485423$
1 Gottesman II, Gould TD: The endophenotype concept in psychiatry: etymology and strategic intentions. Am J Psychiatry 2003;160: 636-645.

2 Meyer-Lindenberg A, Weinberger DR: Intermediate phenotypes and genetic mechanisms of psychiatric disorders. Nat Rev Neurosci 2006;7:818-827.

3 Hobert O: Behavioral plasticity in C. elegans: paradigms, circuits, genes. J Neurobiol 2003; 54:203-223. 
4 Dwyer DS, Donohoe D, Lu X-H, Aamodt EJ: Mechanistic connections between glucose/ lipid disturbances and weight gain induced by antipsychotic drugs. Int Rev Neurobiol 2005; 65:211-247.

5 Schizophrenia Working Group of the Psychiatric Genomics Consortium: Biological insights from 108 schizophrenia-associated genetic loci. Nature 2014;511:421-427.

6 Ardiel EL, Rankin CH: An elegant mind: learning and memory in Caenorhabditis elegans. Learn Mem 2010;17:191-201.

7 Dexter PM, Caldwell KA, Caldwell GA: A predictable worm: application of Caenorhabditis elegans for mechanistic investigation of movement disorders. Neurotherapeutics 2012;9:393-404.

8 Engleman EA, Katner SN, Neal-Beliveau BS: Caenorhabditis elegans as a model to study the molecular and genetic mechanisms of drug addiction. Prog Mol Biol Transl Sci 2016;137: 229-252.

9 Dwyer DS, Awatramani P, Thakur R, Seeni R, Aamodt EJ: Social feeding in Caenorhabditis elegans is modulated by antipsychotic drugs and calmodulin and may serve as a protophenotype for asociality. Neuropharmacology 2015;92:56-62.

10 Anderson DJ, Adolphs R: A framework for studying emotions across species. Cell 2014; 157:187-200.

11 Darwin C: The Expression of the Emotions in Man and Animals. London, Murray, 1872.

12 Insel T, Cuthbert B, Garvey M, Heinssen R, Pine DS, Quinn K, Sanislow C, Wang P: Research Domain Criteria (RDoC): toward a new classification framework for research on mental disorders. Am J Psychiatry 2010;167: 748-751.

13 Cuthbert BN: The RDoC framework: facilitating transition from ICD/DSM to dimensional approaches that integrate neuroscience and psychopathology. World Psychiatry 2014;13:28-35.

14 Liemburg E, Castelein S, Stewart R, van der Gaag M, Aleman A, Knegtering H; Genetic Risk of Outcome Psychosis (GROUP) Investigators: Two subdomains of negative symptoms in psychotic disorders: established and confirmed in two large cohorts. J Psychiatr Res 2013;47:718-725.

15 Kahn RS, Linszen DH, van Os J, Wiersma D, Breggeman R, Cahn W, de Haan L, Krabbendam L, Myin-Germeys I: The association between social anhedonia, withdrawal and psychotic experiences in general and high-risk populations. Schizophr Res 2012;138:290294.

16 Matheson SL, Vijayan H, Dickson H, Shepherd AM, Carr VJ, Laurens KR: Systematic meta-analysis of childhood social withdrawal in schizophrenia, and comparison with data from at-risk children aged 9-14 years. J Psychiatr Res 2013;47:1061-1068.
17 Sokolowski MB: Social interactions in "simple" model systems. Neuron 2010;65:780794.

18 de Bono M, Bargmann CI: Natural variation in a neuropeptide $\mathrm{Y}$ receptor homolog modifies social behavior and food response in $C$. elegans. Cell 1998;94:679-689.

19 de Bono M, Tobin DM, Davis MW, Avery L, Bargmann CI: Social feeding in Caenorhabditis elegans is induced by neurons that detect aversive stimuli. Nature 2002;419:899-903.

20 Frederiksen SO, Ekman R, Gottfries CG, Widerloy E, Jonsson S: Reduced concentrations of galanin, arginine vasopressin, neuropeptide $Y$ and peptide $Y Y$ in the temporal cortex but not in the hypothalamus of brains from schizophrenics. Acta Psychiatr Scand 1991;83:273-277.

21 Karl T, Chesworth R, Duffy L, Herzog H: Schizophrenia-relevant behaviours in a genetic mouse model for Y2 deficiency. Behav Brain Res 2010;207:434-440.

22 Stålberg G, Ekselius L, Lindstrom LH, Larhammar D, Bodén R: Neuropeptide Y, social function and long-term outcome in schizophrenia. Schizophr Res 2014;156:223-227.

23 Kask A, Rago L, Harro J: Anxiolytic-like effect of neuropeptide Y (NPY) and NPY13-36 microinjected into vicinity of locus coeruleus in rats. Brain Res 1998;788:345-348.

24 Sajdyk TJ, Schober DA, Gelhert DR: Neuropeptide $\mathrm{Y}$ receptor subtypes in the basolateral nucleus of the amygdala modulate anxiogenic responses in rats. Neuropharmacology 2002; 43:1165-1172.

25 Gray JM, Karow DS, Lu H, Chang AJ, Chang JS, Ellis RE, Marietta MA, Bargmann CI: Oxygen sensation and social feeding mediated by a C. elegans guanylate cyclase homologue. $\mathrm{Na}$ ture 2004;430:317-322.

26 Hasler G, Drevets WC, Manji HK, Charney DS: Discovering endophenotypes for major depression. Neuropsychopharmacology 2004;29:1765-1781.

27 Padrao G, Mallorqui A, Cucurell D, MarcoPallares J, Rodriguez-Fornells A: Neurophysiological differences in reward processing in anhedonics. Cogn Affect Behav Neurosci 2013;13:102-115.

28 Foussias G, Remington G: Negative symptoms in schizophrenia: avolition and Occam's razor. Schizophr Bull 2010;36:359-369.

29 Foussias G, Agid O, Fervaha G, Remington G: Negative symptoms of schizophrenia: clinical features, relevance to real world functioning and specificity versus other CNS disorders. Eur Neuropsychopharmacol 2014;24:693709.

30 Dagenhardt J, Trinh A, Sumner H, Scott J, Aamodt E, Dwyer DS: Insulin signaling deficiency produces immobility in C. elegans that models diminished motivation states in man and responds to antidepressants. Mol Neuropsychiatry 2017;3:97-107.

31 Moskowitz AK: Scared stiff: catatonia as an evolutionary-based fear response. Psychol Rev 2004;111:984-1002.
32 Porsolt RD, Anton G, Blavet N, Jalfre M: Behavioural despair in rats: a new model sensitive to antidepressant treatments. Eur J Pharmacol 1978;47:379-391.

33 Jaffe RJ, Novakovic V, Peselow ED: Scopolamine as an antidepressant: a systematic review. Clin Neuropharmacol 2013;36:24-26.

34 Greenway SE, Pack AT, Greenway FL: Treatment of depression with cyproheptadine. Pharmacotherapy 1995;15:357-360.

35 Bersani G, Pozzi F, Marini S, Grispini A, Pasini A, Ciani N: 5-HT2 receptor antagonism in dysthymic disorder: a double-blind placebo-controlled study with ritanserin. Acta Psychiatr Scand 1991;83:244-248.

36 Gavard JA, Lustman PJ, Clouse RE: Prevalence of depression in adults with diabetes. Diabetes Care 1993;16:1167-1178.

37 Gupta D, Kurhe Y, Radhakrishnan M: Antidepressant effects of insulin in streptozotocin induced diabetic mice: modulation of brain serotonin system. Physiol Behav 2014;129: 73-78.

38 Powell SB, Zhou X, Geyer MA: Prepulse inhibition and genetic mouse models of schizophrenia. Behav Brain Res 2009;204:282-294.

39 Swerdlow NR, Braff DL, Geyer MA: Sensorimotor gating of the startle reflex: what we said 25 years ago, what has happened since then, and what comes next. J Psychopharmacol 2016;30:1072-1081.

40 Koch M, Schnitzler H-U: The acoustic startle response in rats - circuits mediating evocation, inhibition and potentiation. Behav Brain Res 1997;89:35-49.

41 Howard R, Ford R: From the jumping Frenchmen of Maine to post-traumatic stress disorder: the startle response in neuropsychiatry. Psychol Med 1992;22:695-707.

42 Keane J, Avery L: Mechanosensory inputs influence Caenorhabditis elegans pharyngeal activity via ivermectin sensitivity genes. Genetics 2003;164:153-162.

43 Shiang R, Ryan SG, Zhu YZ, Hahn AF, O'Connell P, Wasmuth JJ: Mutations in the alpha 1 subunit of the inhibitor glycine receptor cause the dominant neurologic disorder, hyperekplexia. Nat Genet 1993;5:351-358.

44 Deutch AY, Goldstein M, Baldino F Jr, Roth RH: Telencephalic projections of the A8 dopamine cell group. Ann NY Acad Sci 1988; 537:27-50

45 Bayer VE, Pickel VM: Ultrastructural localization of tyrosine hydroxylase in the rat ventral tegmental area: relationship between immunolabeling density and neuronal associations. J Neurosci 1990;10:2996-3013.

46 Roussos P, Giakoumaki SG, Pavlakis S, Bitsios $\mathrm{P}$ : Planning, decision-making and the COMT rs4818 polymorphism in healthy males. Neuropsychologia 2008;46:757-763.

47 Chen BT, Patel JC, Moran KA, Rice ME: Differential calcium dependence of axonal versus somatodendritic dopamine release, with characteristics of both in the ventral tegmental area. Front Syst Neurosci 2011;5:39. 
48 Kramer JM, Davidge JT, Lockyer JM, Staveley BE: Expression of Drosophila FOXO regulates growth and can phenocopy starvation. BMC Dev Biol 2003;3:1-14.

49 Dwyer DS, Donohoe D, Aamodt EA: Insulin/ IGF-1 signaling in C. elegans regulates foraging and feeding. Soc Neurosci Abstr 2009; 275.18.

50 Dwyer DS, Horton RY, Aamodt EJ: Role of the evolutionarily conserved starvation response in anorexia nervosa. Mol Psychiatry 2011;16:595-603.

51 Guisinger S: Adapted to flee famine: adding an evolutionary perspective to anorexia nervosa. Psychol Rev 2003;110:745-761.

52 Dwyer DS: Coordinating evolutionarily-conserved response of muscle and brain to optimize performance during starvation; in Preedy VR, Patel VB (eds): Handbook of Famine, Starvation, and Nutrient Deprivation. Berlin, Springer, in press.
53 Pawlowski GJ: Cyproheptadine: weight-gain and appetite stimulation in essential anorexic adults. Curr Ther Res Clin Exp 1975;18:673678.

54 Wirshing DA, Wirshing WC, Kysar L, Berisford MA, Goldstein D, Pashdag J, Minyz J, Marder SR: Novel antipsychotics: comparison of weight gain liabilities. J Clin Psychiatry 1999;60:358-363.

55 Dwyer DS, Aamodt E, Cohen B, Buttner E: Drug elucidation: invertebrate genetics sheds new light on the molecular targets of CNS drugs. Front Pharmacol 2014;5:177.
56 Karmacharya R, Lynn SK, DeMarco S, Ortiz A, Wang X, Lundy MY, Xie Z, Cohen BM, Miller GM, Buttner EA: Behavioral effects of clozapine: involvement of trace amine pathways in C. elegans and M. musculus. Brain Res 2011;1393:91-99.

57 Karmacharya R, Sliwoski GR, Lundy MY, Suckow RF, Cohen BM, Buttner EA: Clozapine interaction with phosphatidyl inositol 3-kinase (PI3K)/insulin-signaling pathway in Caenorhabditis elegans. Neuropsychopharmacology 2009;34:1968-1978.

58 Weeks K, Dwyer DS, Aamodt E: Antipsychotic drugs activate the $C$. elegans Akt pathway via the DAF-2 insulin/IGF-1 receptor. ACS Chem Neurosci 2010;1:463-473.

59 Weeks K, Dwyer DS, Aamodt EJ: Clozapine and lithium require Caenorhabditis elegans $\beta$-arrestin and serum- and glucocorticoid-inducible kinase to affect DAF-16 (FOXO) localization. J Neurosci Res 2011;89:1658-1665. 